tained at the temperature of liquid nitrogen are now believed to be stable, more or less indefinitely, so that viable cultures preserved in this way are preferable in the interests of reproducibility. The meeting recom. mended that reliable methods for the cryogenic preservation of algae be developed and adopted as soon as they are available.

\section{New Ways in the Infrared}

\section{from our Molecular Biology Correspondent}

THe study of protein conformations in solution has in recent years become harder. Except perhaps for proteins containing a high proportion of $\alpha$-helix, interpretation of optical rotatory dispersion and circular dichroism has become increasingly complicated, chiefly because of the discovery that various side chains can contribute sizable Cotton effects of their own. This uncertainty has made the development of other methods of observing polypeptide chain conformation and its changes increasingly desirable. The recent resurgence of infrared spectroscopy of aqueous solutions is therefore to be welcomed and, as will be seen, it has already produced some promising results.

The new work is by Susi, Timasheff and Stevens (J. Biol. Chem., 242, 5460; 1967), who have looked at the infrared spectra, primarily in $\mathrm{D}_{2} \mathrm{O}$ solution, of several synthetic polypeptides and proteins. It is already well known that the so-called amide I frequency of the polypeptide chain is conformationsensitive, and that the formation of a pleated-sheet $(\beta)$ structure causes a considerable shift to lower frequency. A less familiar observation, which was recently reported by the same group of workers, is the distinct, though smaller, shift which occurs when the chain enters the a-helical conformation. Thus, it is shown that poly-Llysine in $\mathrm{D}_{2} \mathrm{O}$ solution can exhibit an amide $\mathrm{I}$ band at $1,635,1,645$ or $1,611 \mathrm{~cm}^{-1}$, depending on whether it is in the random, the a-helical, or the antiparallel pleatedsheet state. In the latter form there is also a characteristic weak component, due to another vibrational mode, at $1,680 \mathrm{~cm}^{-1}$. ( $\mathrm{In} \mathrm{H}_{2} \mathrm{O}$ solution there are corresponding, though displaced, frequencies, but experimental diffculties generated by the high absorption of the solvent in this range are prohibitive.) These band positions may vary somewhat from molecule to molecule, and will depend, for example, on the strength of hydrogen bonding. In proteins then a certain spread of frequencies is found for each conformation, but this does not appear to be sufficient to cause any serious ambiguity.

In a second article Timasheff et al. (J. Biol. Chem., $242,5467 ; 1967)$ survey a large number of proteins. In the majority of cases the peak of the amide I band lies either at about 1,650 or near $1,637 \mathrm{~cm}^{-1}$. The first of these groups comprises those proteins known to have a substantial proportion of $\alpha$-helical structure-as myoglobin and lysozyme, for example. The second consists of proteins known or thought to contain little $\alpha$-helical, or other regular, structure. In almost all cases, however, prominent shoulders are observed which reveal the amide I band to be complex. Thus in lysozyme, the inflexion at $1,632 \mathrm{~cm}^{-1}$ must reflect the $\beta$-structure known to be present, and in many cases shoulders at $1,685 \mathrm{~cm}^{-1}$ also indicate the presence of antiparallel $\beta$-forms. Indeed, in all cases for which erystallographic data are available, the conformation is qualitatively matched by the distribution of amide I frequencies. In a few instances partial resolution of components of the band maximum is observed.

Some striking effects engendered by denaturation are shown. Insulin, for example, has been converted into three different denatured states: in methanol solution, the position of the band-peak is unchanged, thus showing that the $\alpha$-helical structure persists; in alkaline solution it shifts to a position characteristic of a random coil, and on heating at low $p \mathrm{H}$ antiparallel $\beta$-sheets appear. These effects are paralleled by changes in the ultraviolet Cotton effects. Again, $\beta$-lactoglobulin in the native state evidently contains a large proportion of $\beta$-structure; addition of methanol leads to denaturation with progressive formation of $a$-helix, though a different $\beta$-structure, associated with aggregation, also appears. Again the transition follows optical rotatory dispersion changes. The utility of this approach is obvious: it will assuredly find wider use.

\section{Algae on Film \\ by our own Correspondent}

"Micro-ALGaE-a Study of Movement and Multiplication" is the title of a film shown to the Linnean Society at a meeting on January 18. The film, which is intended primarily as a teaching aid, was made by Dr P. E. Brandham of the Royal Botanic Gardens, Kew.

The film is silent, with explanatory captions, and in the twenty-five minutes for which it runs shows some very active fresh water algae. Dr Brandham minimized vibration, usually a big problem in cine-microphotography, by standing the microscope on a rubber pad and a sprung base plate. For the slower movers and for showing cell division, time lapse photography was used, with apparent accelerations of from 30 to 3,840 times.

First, flagellar action is shown in a dinoflagellate, Ceratium, and in two euglenoid and three volvocacean genera. Dark field microscopy shows the flagella particularly clearly, and in the water around Volvox small particles can be seen moving as a result of flagellar action. Phacus is seen moving by its characteristic rolling motion, and looking like a sting ray doing contortions. Euglena gives a perfect display of euglenoid movement.

Time lapse photography makes it possible to see blue-green algae, including Anabaena, gliding around, and, among the Chlorophyta, the very slow movement which brings filaments of Spirogyra together for conjugation can be seen. Diatoms are shown shunting to and fro-the ease with which they can change direction is clearly seen. The desmid Closterium acerosum moves by a form of jet propulsion, by secreting mucilage from one end of its thin body, like toothpaste from a tube. The mucilage hardons and Closterium is pushed forward. There are also some fine time lapse pictures of cell division in several genera, including colonial algae and desmids.

\section{Crystalline Ribosomes}

\section{from our Cell Biology Correspondent}

THE spatial organization of RNA and proteins in ribosomes will remain unknown until somebody manages to produce crystalline ribosomes which ean be 